\title{
Remesas y migración en el municipio de Tecamachalco Puebla, México
}

\section{Remitaces and migration in the municipality of Tecamachalco Puebla, México}

\author{
XIMITL-ISLAS, Iván*†, RODRÍGUEZ-DE LA VEGA, Marisol, CABILDO-OREA, Alejandra y \\ MACHORRO-DÍAZ, Rafael
}

Universidad Tecnológica de Tecamachalco, Avenida Universidad N 1, Colonia La Villita, C.P. 75483.

ID $1^{\text {er }}$ Autor: Iván, Ximitl-Islas / ORC ID: 0000-0002-6525-5106, Researcher ID Thomson: S-7952-2018, CVU CONACYT ID: 168266

ID $1^{\text {er }}$ Coautor: Marisol, Rodríguez-De La Vega / ORC ID: 0000-0002-0263-4504, Researcher ID Thomson: S-7774- 2018, CVU CONACYT ID: 424632

ID $2^{\text {do }}$ Coautor: Alejandra, Cabildo-Orea / ORC ID: 0000-0002-6088-5707, Researcher ID Thomson: S-7798-2018, CVU CONACYT ID: 947359

ID $3^{\text {er }}$ Coautor: Rafael, Machorro-Díaz / ORC ID: 0000-0002-1776-7174, Researcher ID Thomson: S-7910-2018, CVU CONACYT ID: 947608

DOI: $10.35429 / J T M S .2019 .16 .5 .1 .15$

Recibido 04 de Junio 2019; Aceptado 30 Septiembre, 2019

\section{Resumen}

En esta oportunidad se presentan los resultados de una encuesta aplicada en el municipio de Tecamachalco, Puebla, México en la cual la población objetivo se centra en las familias con una o más personas han migrado hacia los Estados Unidos. La metodología se basa en la recopilación de información financiera de las remesas familiares por entidad federativa con datos del BANXICO (Banco de México) y en la aplicación de 478 cuestionarios en un trabajo de campo. También se consultó el Índice de Intensidad Migratoria CONAPO (Consejo Nacional de Población), para analizar el número de familias en Tecamachalco que reciben remesas.La contribución de este proyecto radica en la información que se obtuvo en el fenómeno de la migración laboral en el estado de Puebla, que ocupa el sexto lugar en recepción de remesas familiares y cuyoprincipal mercado laboral en los Estados Unidos se lleva a cabo en el Sector de Servicios primordialmente. Los estados donde residen principalmente los migrantes de Tecamachalco son: California, Texas y Nueva York, y las ciudades fronterizas con mayor recurrencia son: Tijuana, Nogales, Agua Prieta, Nuevo Laredo, Reynosa y Piedras Negras. Del mismo modo, también se obtuvo información del impacto que tienen las remesas así como su empleo y forma de envío.

Migración, Remesas, Frontera

\begin{abstract}
This time, the results of a survey applied in the Municipality of Tecamachalco, Puebla, Mexico, in which the target population is focused on families with one or more people who have migrated to the United States, are presented. The methodology is based on the collection of financial information on family remittances per Federal Entity with data from BANXICO (Bank of Mexico) and on the application of 478 questionnaires in a fieldwork. Also, the Migration Intensity Index was consulted at the National Population Council (CONAPO for its initials in Spanish) to analyze the number of families in Tecamachalco that receive remittances. The contribution of this project lies in the information obtained in the phenomenon of labor migration in the state of Puebla, which ranks sixth in receiving family remittances and whose main labor market in the United States takes place in the Service Sector primarily. The states where Tecamachalco migrants mainly reside are: California, Texas, and New York, and the border cities with the greatest recurrence are: Tijuana, Nogales, Agua Prieta, Nuevo Laredo, Reynosa, and Piedras Negras. Similarly, information was also obtained on the impact of remittances as well as their use and shipping method.
\end{abstract}

Migration, Remittances, Frontier

Citación: XIMITL-ISLAS, Iván, RODRÍGUEZ-DE LA VEGA, Marisol, CABILDO-OREA, Alejandra y MACHORRODÍAZ, Rafael. Remesas y migración en el municipio de Tecamachalco Puebla, México. Revista Transdisciplinaria de Estudios Migratorios. 2019, 5-16: 1-15

\footnotetext{
* Correspondencia al Autor (correo electrónico: ivanximitl@ hotmail.com)

$\dagger$ Investigador contribuyendo como primer Autor.
} 


\section{Introducción}

Para contextualizar el fenómeno de la migración entre México y los Estados Unidos es necesario establecer que los límites territoriales actuales fueron definidos desde el siglo XIX, el territorio que fue cedido a los Estados Unidos de Norteamérica, lo importante es que esta decisión ha tenido repercusiones hasta nuestros días en pleno siglo XXI. El establecimiento de la frontera norte de México, determinó un activo fijo como lo es la tierra, el proceso de la acumulación del capital tiene su inicio en la invasión norteamericana de 1846, y posteriormente tanto la negociación del Tratado de Guadalupe Hidalgo, la frontera norte de México con Estados Unidos, a partir de 1848, gran parte de nuestro territorio nacional fue transferido por el presidente Antonio López de Santa Anna al gobierno Estadounidense (más de la mitad del territorio).

La frontera natural que es el río Bravo, (que ya se había establecido desde los códices prehispánicos), delimitando una frontera física entre los mexicanos que quedaron fuera del territorio estadounidense y los que quedaron dentro, esta separación como ha sucedido en distintos países en diferentes épocas, se presentó una fractura entre las relaciones establecidas entre las familias de mexicanos que fueron los precursores del establecimiento de nuevas ciudades en los Estados Unidos, esta es una de las razones por que los territorios de California, Arizona, Nuevo México y Texas, actualmente donde residen la mayor parte de indocumentados se encuentran en dichos estados de la Unión Americana, es decir que la acumulación originaria de capital de los Estados Unidos inicia en esa parte de territorio.

Al haber erradicado a la población de los indios del norte (los sobrevivientes fueron remitidos a reservas) y apropiándose de los recursos mineros, la "franja de oro" los recursos en hidrocarburos y la fuerza de trabajo en minería, manufacturas, construcción de obras civiles, ferrocarriles y mano de obra para las cosechas, incluso han participado en ejércitos para luchar en la primera y segunda guerra mundial defendiendo a los Estados Unidos a cambio de la nacionalidad.

\footnotetext{
${ }^{1}$ Lourdes G. García "La protección del trabajador indocumentado, las legislaciones mexicanas y estadounidense", Tesis, UNAM, México, 1992, p. 3.

\begin{abstract}
Estas manifestaciones permitieron la adaptación cultural de los nuevos estadounidenses legales y los ilegales con raíces mexicanas para forjar el imperio que es actualmente los Estados Unidos de Norteamérica. Históricamente México y los Estados Unidos han participado en una relación bilateral de dependencia con el país vecino del norte, ha sido la acumulación originaria del capital, desde mediados del siglo XIX, el proceso de Independencia $(1810$ - 1821), la invasión norteamericana (1846 - 1848) y la invasión francesa (1862) fueron cruciales en la nueva conformación geopolítica para nuestro país, las leyes de Reforma (1857) fincaron la estructura de un sistema capitalista emergente, en Europa la revolución industrial fue determinante en la industrialización y la introducción del ferrocarril en México fueron las bases de una red de comunicaciones durante la segunda mitad del siglo XIX. Por otra parte, Canadá y Estados Unidos, han sido socios comerciales.
\end{abstract}

Las discrepancias que se presentan en las relaciones internacionales entre México y los Estados Unidos, inician desde mediados del siglo XIX, la fuerza de trabajo que demandaba una nación como los Estados Unidos, el problema de la migración no es un asunto nuevo en las agendas en materia de relaciones internacionales de ambos países, uno de los tratados internacionales que definiría los límites territoriales el Tratado de Guadalupe Hidalgo, En su Tesis de Lourdes García expone:

"Para hablar de trabajadores mexicanos en Estados Unidos es preciso remontarse al año de 1848 cuando se firmó el tratado de Guadalupe Hidalgo, en el cuál México perdió gran parte de su territorio. En esa ocasión más de 73 mil ciudadanos mexicanos quedaron varados en el nuevo territorio estadounidense. En ese entonces, el gobierno de Estados Unidos otorgó un plazo de un año para que estos mexicanos abandonaran el territorio o bien cambiaran de nacionalidad", 1 
La migración hacia los Estados Unidos parte desde un acuerdo internacional, gran parte del territorio mexicano quedó en manos del gobierno estadounidense esto corresponde a la región sudoeste primera fase, esta zona la constituyen básicamente los estados que son frontera sur con México y que antes de la firma de dicho acuerdo perteneció a los territorios de California, Arizona, Nuevo México y Texas. La migración internacional hacia los Estados Unidos se ha desarrollado en diferentes etapas, el punto de partida en el análisis de este proceso histórico, la acumulación de capital de los Estados Unidos inicia desde la invasión a México en 1846 hasta la culminación con el Tratado de Guadalupe Hidalgo, esto fue el cimiento de una estructura para que el sistema capitalista de producción tuviera su acumulación originara de capital, en donde uno de los factores de la producción como lo es el activo fijo que es la tierra, por lo que en poco más de siglo y medio las transformaciones políticas, económicas y sociales que ha sufrido el vecino país del norte ha influido en la determinación de la estructura económica de México, para comprender la migración internacional contemporánea, ante la ratificación del TMEC antes TLC ( Tratado de Libre Comercio) entre Estados Unidos, Canadá y México puesto en marcha desde el 1 de enero de 1994 y cuáles han sido sus efectos.

\section{Marco Teórico}

La migración internacional no se puede ver desde la perspectivita individual para mejorar las condiciones de vida, debido a que es un proceso de integración en otro contexto, dejando sus raíces para adaptarse a otro estilo de vida, condiciones laborales, el migrante se tiene que adaptar a un nuevo escenario, esto puede trascender no sólo en un migrante sino las generaciones futuras también participan, por lo que este proceso es largo y también puede ser generacional. Sthephen Castles y Mark J. Miller, en su libro "La era de la migración, movimientos internacionales de población en el mundo moderno" nos exponen un enfoque alternativo para la comprensión del fenómeno migratorio:

\footnotetext{
${ }^{2}$ Sthephen Castles y Mark J. Miller, "La era de la migración, movimientos internacionales de población en el mundo moderno" Editorial Porrúa, UAZ, Cámara de Diputados, México 2004, p.14

"Los movimientos adoptan muchas formas: las personas emigran como obreros, especialistas altamente calificados, empresarios, refugiados o como parientes de migrantes previos. Independientemente de si la intención original es un movimiento temporal o permanentemente, muchos se establecen de manera definitiva. Se crean redes de migrantes que vinculan las áreas de origen y destino y ayudan a impulsar cambios importantes en las dos. Las migraciones pueden cambiar las estructuras demográficas, económicas y sociales y conllevar una nueva diversidad cultural, lo que con frecuencia cuestiona su identidad nacional." 2

Castles y Miler, clasifican en 5 ejes en torno al fenómeno migratorio:

1. La globalización de la migración: La tendencia de a que cada vez más países se vean afectados críticamente en la forma simultánea por los movimientos migratorios. Además de la diversidad de las áreas de origen también se incrementa, de modo que la mayor parte de los países de inmigración reciben a quienes provienen de un amplio espectro de antecedentes económicos, sociales y culturales.

2. La aceleración de la migración: en la actualidad los movimientos internacionales de personas incrementan $\mathrm{su}$ volumen en todas las regiones importantes. Este incremento hace que aumenten tanto la urgencia como las dificultades de las políticas gubernamentales.

3. La diferenciación de la migración: la mayoría de los países no tiene una sola clase de inmigración, como la del tipo laboral, de refugiados, o de quienes se establecen de forma permanente; sino que presentan al mismo tiempo toda una gama. Es característico de las cadenas migratorias que se inician con un tipo de movimiento y frecuentemente con otras formas, a pesar (o a veces por eso) de los esfuerzos del gobierno por detener o controlar un movimiento. 
Esta diferenciación presenta un obstáculo mayor par las medidas políticas nacionales e internacionales.

4. La feminización de la migración: Las mujeres juegan un papel importante en todas las regiones y en la mayor parte (no en todos) de los tipos de migración. En el pasado, las migraciones laborales $\mathrm{y}$ muchos de los movimientos de refugiados tenían dominio masculino y con frecuencia las mujeres eran tratadas bajo la categoría de reunificación familiar. Desde los sesenta las mujeres han desempeñado un papel importante en la migración laboral.

5. La creciente politización de la migración: La política doméstica, las relaciones bilaterales y regionales y las políticas de seguridad nacional de los estados en el mundo se ven afectadas cada vez más por la migración internacional.

Dentro del marco del Tratado de Libre Comercio la migración laboral fue un rubro que no fue considerado, en este sentido la Nueva Economía de la Migración Laboral es una teoría contemporánea que nos explica la situación en un contexto de mercado laboral, Stark es uno de los principales teóricos contemporáneos que explica el comportamiento de la migración, uno de los aspectos de la migración, se basa en las decisiones, no sólo del migrante sino a un grupo que no participan de manera directa, por lo que expresa:

"Las decisiones de emigrar las toman a menudo conjuntamente el migrante y algún grupo de no migrantes. Costes y ganancias se comparten, especificándose la norma que rige la distribución de ambas cosas en un convenio contractual implícito entre las dos partes. Por ejemplo, un componente importante de los rendimientos directos que obtiene la familia no migrante procedentes de la migración de un miembro que de ella son las remesas que envía dicho miembro." 3
Es decir, lo que sugiere la teoría es que existe una ventaja comparativa de la familia partir de las remesas, como una entidad que se divide entre los miembros que la integran y sobre todo los jóvenes que se encuentran en edad de trabajar, debido a ser la fuerza de trabajo, se concibe a la migración como una estrategia "calculada "de un grupo, por lo que asegura lo siguiente:

\section{"La naturaleza de la interacción} dentro del grupo podría contribuir también a explicar algunas de las características del comportamiento económico, de los migrantes, para empezar, los migrantes superan a menudo el rendimiento de los nativos de la economía acogida. Además, una fuerte dependencia del "capital de redes y de parientes" es otra característica destacada de los modelos de comportamiento de los migrantes. [...] Este resultado da una explicación interesante de la observación según la cual los nuevos migrantes los ayudan aquellos que emigraron antes; una buena forma de conseguir una elevada proporción de los intercambios se realice entre migrantes, cuando estos sean pocos, es que vengan más. La llegada de nuevos migrantes proporciona nuevos beneficios a los que vinieron primero. Indica también una solución de la aparente incoherencia del comportamiento altruista dentro de un grupo pequeño (la familia) y el comportamiento egoísta de grandes grupos (por ejemplo, el mercado)." 4

Las remesas suelen ser como “multiplicador de la inversión”, debido a que las transacciones de las remesas familiares, estimulan el ahorro y la inversión, John Mynard Keynes en su obra "Teoría general de la ocupación el interés y el dinero" nos señala que el estado puede proponer el pleno empleo, pero debido a la privatización de las empresas paraestatales, el estado ha dejado que la oferta del trabajo sea regulada por las fuerzas del mercado laboral, en la división internacional del trabajo.

\footnotetext{
${ }^{3}$ Stark Oded, "La migración del trabajo", Ministerio del trabajo y Seguridad Social, España, Madrid España, 1993, p. 40 .
}

${ }^{4}$ John Maynard Keynes, "Teoría general de la ocupación el interés y el dinero", Fondo de Cultura Económica, México, 2013, pp. 134-135. 
Por otra parte John Maynard Keynes, establece que para una sociedad moderna debe de tener mayor cuidado en los siguientes factores en la propensión marginal al consumir y el multiplicador de la inversión:

"1). El método al financiar la política, y el mayor volumen que impone el aumento de ocupación y el alza de los precios que le acompaña, puede tener el efecto de hacer subir la tasa de interés y retardar así la inversión en otras direcciones, a menos que las autoridades monetarias tomen providencias en sentido contrario; en tanto que, al mismo tiempo, la elevación en el costo de bienes de capital reduce la eficacia marginal para el inversionista privado, lo que requerirá una baja real en la tasa de interés para equilibrarla.

2). Con la psicología confusa que con frecuencia priva, el programa de gobierno puede, a través de sus efectos sobre la confianza aumentar la preferencia por la liquidez o disminuir la eficacia marginal del capital, lo que, a su vez, puede retardar otras inversiones, a menos que se tomen medidas para evitarlo.

3). En un sistema abierto, con relaciones de comercio exterior, parte del multiplicador de la inversión acrecentada beneficiará a la ocupación en países extranjeros, ya que cierta proporción del aumento en el consumo reducirá la balanza exterior favorable de nuestro propio país; de manera que si consideramos solamente el efecto sobre la ocupación nacional, por oposición a la mundial, tenemos que reducir la cifra del multiplicador. Por otra parte, nuestro país puede recobrar esta merma mediante repercusiones favorables debidas a la acción del multiplicador en los países extranjeros, al aumentar su actividad económica. $" 5$

Por otra parte, Duran y Massey en 2007, nos indican la incorporación de la fuerza de trabajo mexicana en los EUA, la incorporación de mano de obra mexicana a los mercados laborales, con trabajos poco calificados y a un precio menor de la media en comparación con el pago del salario de trabajadores indocumentados, sin capacitación para el trabajo, sin gastos médicos, seguros, etc.

\footnotetext{
${ }^{5}$ Ibídem, p. 41-42.

${ }^{6}$ Duran Jorge y Douglas Massey "Clandestinos, Migración Estados Unidos en los albores del siglo XXI" Universidad

Los salarios no sólo reflejan las condiciones de oferta y demanda, sino que también representan estatus y prestigio, estos aspectos sociales son inherentes al tipo de trabajo y a los diferentes salarios, lo cual indican Duran y Massey:

"La mayoría de los migrantes se inicia como posibles asalariados que buscan ganar dinero para un objetivo específico que les permita mejorar su situación o bienestar en su lugar de origen - construir una casa, pagar la escuela, comprar tierra, adquirir bienes de consumo. Por otra parte, la diferencia entre los estándares de vida entre las sociedades desarrolladas y las sociedades en desarrollo implica que aun con un salario bajo en el exterior sea aparentemente abundante; aunque un inmigrante sea consciente de que un trabajo en el extranjero es de bajo nivel - lavar platos o limpiar baños-, él no se considera como a sí mismo como parte de la sociedad que los recibe. Más bien se ve como un miembro de su comunidad de origen, en el cual los que trabajan en el exterior y envían remesas tienen un prestigio considerable." 6

Por lo que los autores agregan que existe una dualidad entre el trabajo y el capital, la fuerza de trabajo es un factor de la producción que es variable, cuando la demanda cae con una crisis, guerra, o desastres naturales, (por citar algunos ejemplos) la demanda del mercado laboral se contrae, por lo que los asalariados se ven en la necesidad de absorber los costos del desempleo y subempleo, la fuerza de trabajo de indocumentados toma la forma de un mercado laboral segmentado.

Otro de los puntos importantes a tratar es que la comunidad de migrantes no se encuentra dispersa, sino que se está en una concentración en donde les permite tener una relación con el lugar de origen en donde comparten ciertas afinidades con las aspiraciones de la movilidad social, aunque no es generalizado también existen familias que dejan de tener comunicación con su familia y la comunidad de origen.
Autónoma de Zacatecas y Miguel Ángel Porrúa Editores, México, 2003, p. 19. 
"La concentración significativa de personas de un mismo grupo étnico crea una demanda de productos culturales especializados y servicios que sólo pueden ser satisfechos por empresarios inmigrantes calificados. Además, su acceso privilegiado a un grupo cada vez mayor de inmigrantes de bajos ingresos los coloca en la posición ventajosa frente a empresas de la competencia que está fuera del enclave." 7

La migración mexicana tiene esta característica, debido a que el llamado "mercado de la nostalgia" es un nicho de mercado estratégico para la comunidad mexicana, el mercado de alimentos de origen mexicano: chile, maíz, tortillas, tamales, huitlacoche, etc. así como la demanda de servicios de envío de paquetería, remesas, música, además el efecto multiplicador de la inversión que se genera en su lugar de origen como lo es la construcción, las fiestas patronales, los arreglos florales, los eventos sociales, bodas, XV años, bautizos, graduaciones, son ejemplos mismos de la identidad de la sociedad mexicana.

Estas redes son un proceso que está en expansión y muestran los desequilibrios entre la demanda estructural de trabajadores en el nivel más bajo y la limitada oferta de dichos trabajadores en el nivel doméstico ha generado una demanda creciente de inmigrantes en los países desarrollados. Duran y Massey afirman:

"Las redes migratorias son conjuntos de lazos interpersonales que conectan a los migrantes con otros migrantes que los precedieron y con no migrantes en las zonas de origen y destino mediantes nexos de parentesco, amistad y paisanaje. Estos nexos incrementan la posibilidad de movimiento internacional porque bajan los costos y los riesgos del desplazamiento y aumentan los ingresos netos de la migración. Las conexiones dentro de la red constituyen una forma de capital social de que las personas pueden beneficiarse para acceder a diversas formas de capital financiero: empleo en el extranjero, pago de coyotes, salarios más altos y la posibilidad de hacer ahorros y enviar remesas. El crecimiento de redes verificado a través de la reducción progresiva de los costos puede explicarse teóricamente por la progresiva reducción de riesgos.
Cada nuevo migrante expande la red y reduce los riesgos de movimientos para todos aquellos con quienes está relacionado, eventualmente pueden llegar a hacer que sus movimientos carezcan de riesgos y sea manos difícil diversificar la ubicación laboral de los miembros de su familia". ${ }^{8}$

El fundamento que tiene la red social para promover los desplazamientos internacionales al emplear los vínculos sociales con familiares y amigos que llegaron primero al país donde les brindan alojamiento, incluso les consiguen en algunos casos empleo donde ellos mismos desempeñan, es común encontrar los lazos sociales en el sector de los servicios como el restaurantes, es clásico el parentesco o la amistad o vecindad, por lo que se crean circuitos migratorios con un nodo que es la ciudad o región objetivo en donde la información es tan cotidiana, como saber en cuanto está el salario, cuánto pagan de coyote, la renta, gastos de traslado, entre otros. Las redes de migrantes han aumentado en la participación económica y política, las familias con dos o tres generaciones son el ejemplo de las agrupaciones en nuestro vecino del norte, la identidad que es un factor cultural se muestra en los diversos clubes de migrantes de las diferentes regiones de México, se puede inferir que el problema político bilateral entre Estados Unidos y México (frontera norte de México alrededor de 3,000 $\mathrm{km}$.

\section{Metodología}

En cuanto a información financiera se consultó las bases de datos del Banco de México, en la sección de estadísticas, en la parte de Balanza de pagos con la información de remesas familiares, se consultó del periodo mensual de enero de 1995 a diciembre de 2018, en donde se muestran los gráficos, la información se procesó en tasa de crecimiento, promedios móviles trimestrales, y tendencia de las remesas familiares, también se obtuvo la información estadística por entidad federativa para ubicar dentro del contexto nacional en que ubicación se encuentra el estado de Puebla con relación con el resto de las entidades federativas. 
La ecuación de la recta de las remesas mensuales de 1995 a 2018 fue $\mathrm{y}=2820.6 .1+1210.5 \mathrm{x}$ de con una pendiente positiva y el valor de $\mathrm{R}^{2}=0.8533$. De acuerdo con la metodología de Stevenson, 1981, el cálculo de la ecuación lineal se realiza calculando las variables $\alpha$ y $\beta .{ }^{9}$ Tal como se muestra en la proyección de las remesas.

$$
\begin{aligned}
& y=\alpha+\beta t \\
& y=2820.6+1210.5 x \\
& \beta=\frac{n \sum t Y-\sum t \sum Y}{n \sum t^{2}-\left(\sum t\right)^{2}} \\
& \alpha=\frac{n \sum Y-b \sum t}{n}
\end{aligned}
$$

\section{Proyecciones de las remesas anuales: (Millones de dólares)}

$$
\begin{aligned}
& 2019 \mathrm{y}=2820.6+1210.5(25)=33,083.1 \\
& 2020 \mathrm{y}=2820.6+120.5(26)=34,293.6
\end{aligned}
$$

\section{El diseño de la muestra}

El diseño de la muestra se realizó de acuerdo con la información del Consejo Nacional de Población (CONAPO), mediante e Índice de Intensidad Migratorio, el cuál mide la intensidad migratoria a nivel nacional, estatal y municipal, el cual muestra el número de familias que reciben remesas, para el municipio de Tecamachalco el $2.43 \%$ reciben remesas, el cálculo se realizó con la metodología de Raúl Rojas Soriano, (2013), muestra para estudios sencillos:

$$
\begin{aligned}
& n=\frac{\mathrm{Z}^{2} \mathrm{pq}}{\mathrm{E}^{2}} \\
& \mathrm{n}=\frac{(1.96)^{2}(0.5)(0.5)}{0.05^{2}} \\
& \mathrm{n}=384 \\
& n=\frac{\mathrm{no}}{1+\frac{\mathrm{no}-1}{\mathrm{~N}}} \\
& n=\frac{384}{1+\frac{384-1}{15,402}} \\
& \mathrm{n}=375
\end{aligned}
$$

El tamaño de la muestra es de 375 familias que reciben remesas de 15,402 familias en el municipio de Tecamachalco, debido al impacto de este estudio regional se aplicaron 478 cuestionarios en la encuesta sobre remesas y migración con la ayuda de alumnos de nivel Técnico Superior en Contaduría de la Universidad Tecnológica de Tecamachalco.

El objeto de estudio son las familias que reciben remesas que se presenta en la región de Tecamachalco Puebla, siendo el estado de Puebla uno de los principales receptores de remesas a nivel nacional,

La técnica que se empleó en el trabajo de campo fue la entrevista dirigida a familiares y a migrantes de retorno por medio de un instrumento que es el cuestionario, en el municipio de Tecamachalco Puebla que contaran con información de la trayectoria laboral, así como las rutas de cruce de la frontera norte, los lugares donde residen y trabajan en los EUA, teniendo el principal interés el ingreso gasto, la actividad económica que realizaban en México y la que realizaban en los EUA, así como la actividad principal de su lugar de origen, ver cuáles son las redes que intervienen en el proceso migratorio, las causas de la decisión de migrar para determinar la situación del mercado laboral de la migración laboral ilegal, enel Directorio Estadístico Nacional de Unidades Económicas de INEGI se encontraron 66 establecimientos de servicios financieros, siendo un nodo de desarrollo regional.

\section{Resultados}

Para el año 2018 los ingresos por remesas fueron de 33,470.42 millones de dólares, se presentan los resultados según el Banco de México: Michoacán 3,391.5 m.d.d. (10.1\%), Jalisco 3,287.3 m.d.d. (9.8\%), Guanajuato 3,044.7 m.d.d. (9.1\%), Estado de México 1,902.4 m.d.d. (5.7\%), Oaxaca 1730.5 m.d.d. (5.2\%), Puebla $1,698.3$ m.d.d. (5.1\%), Guerrero 1,614.5 m.d.d. (4.8\%), Ciudad de México 1,414.7 m.d.d. (4.2\%), Veracruz 1,375.7m.d.d. (4.1\%), y el resto de los Estados recibieron el $41.9 \%$, cabe señalar que tan solo la captación de las remesas se concentró en nueve entidades, (el 58.10\%).
${ }^{9}$ Stevenson Wiliam J, "Estadística para administración y economía”, Editorial Harla, México, 1981, p. 500. 
A su vez es notable que la región Centro Occidente siga siendo la principal región que recibe remesas, mientras que la Ciudad de México y el Estado de México aparecen en este registro perteneciendo la zona metropolitana en la recepción de remesas.

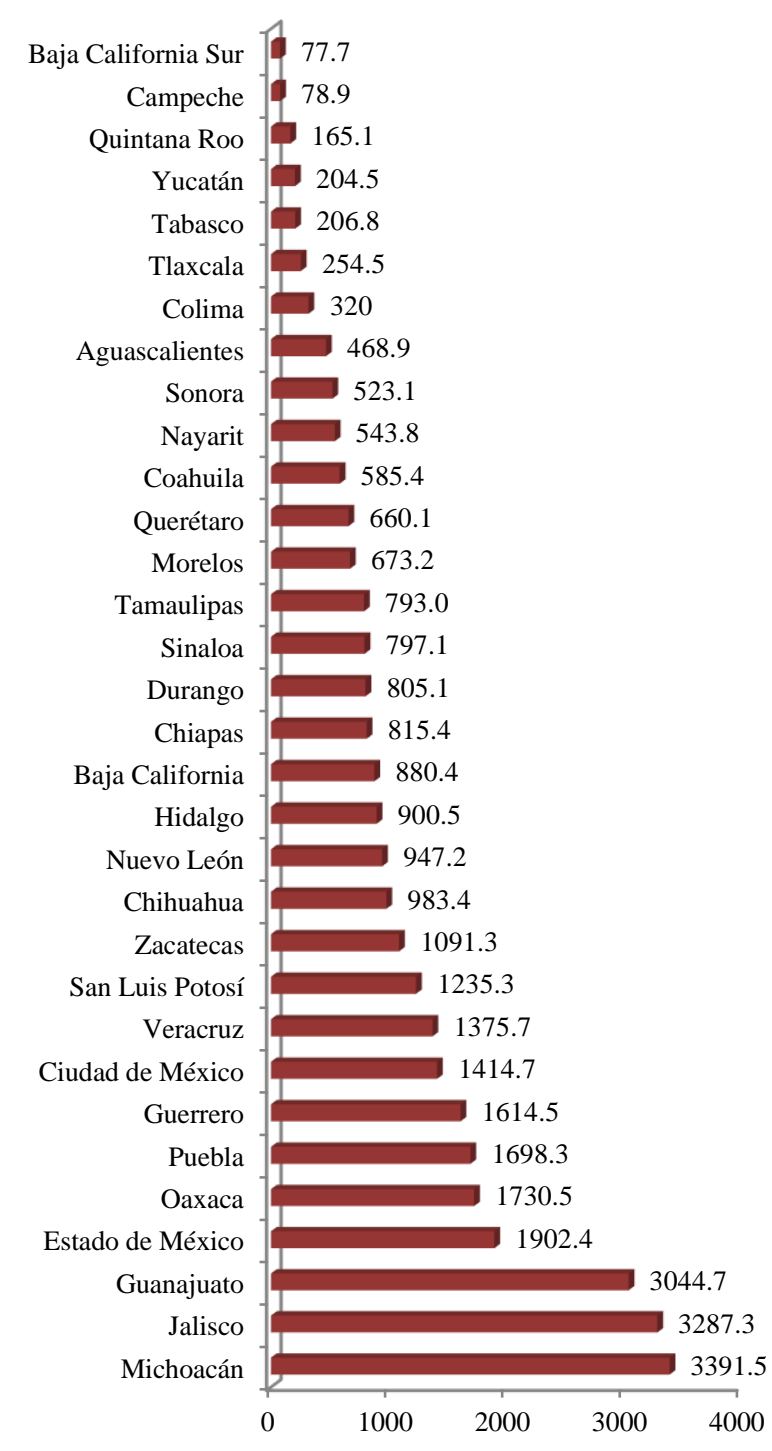

Figura 1 México, remesas familiares 2018, por Entidad Federativa (Millones de dólares)

Fuente: Elaboración propia con datos del Banco de México

En la Fig. 1, se observa que Puebla se encuentra dentro de los diez primeros Estados de la República Mexicana, ocupando el $6^{\circ}$ lugar de los que generaron más remesas familiares en el 2018.

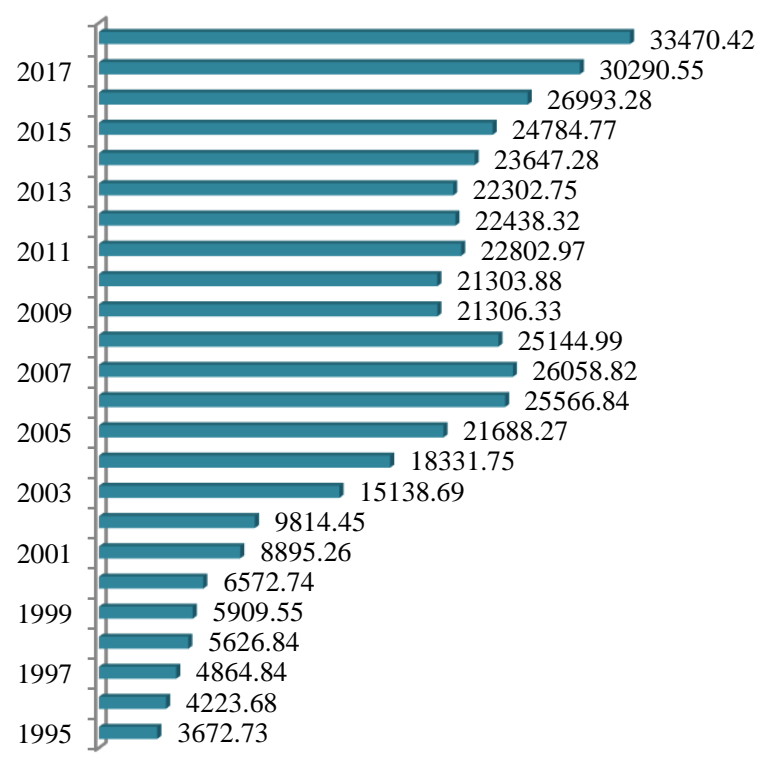

Figura 2 México, remesas familiares 1995 - 2018 (Millones de dólares)

Fuente: Elaboración propia con datos del Banco de México

http://www.banxico.org.mx/SieInternet/consultarDirector ioInternetAction.do? sector $=1 \&$ accion $=$ consultarCuadro Analitico\&idCuadro $=C A 11$ \&locale $=e s$

En la Fig. 2, se denota un crecimiento del año 1995 al 2018 de un $811.4331 \%$, que a pesar de no ser constante, en los últimos tres años se ha visto un incremento importante en la recepción de remesas familiares a México.

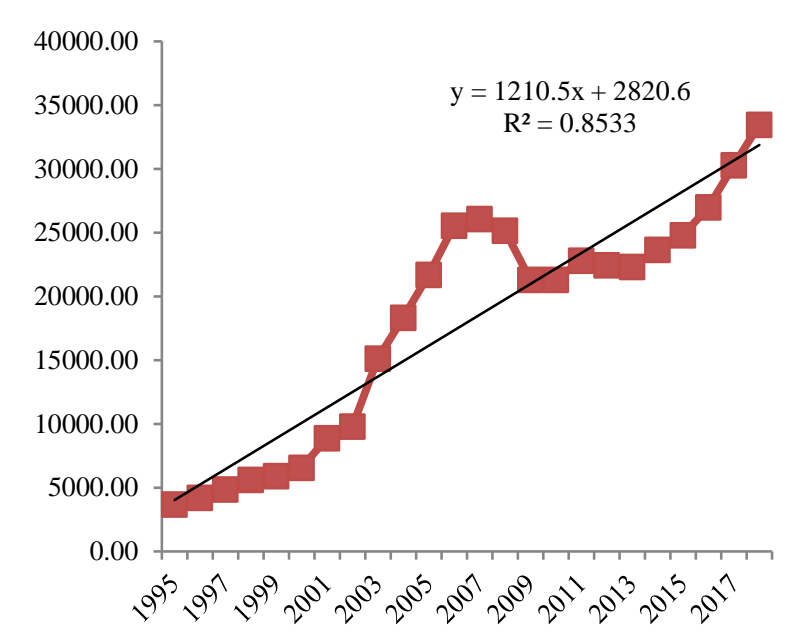

Figura 3 México, remesas familiares 1995-2018 (Millones de dólares) Regresión Lineal

Fuente: Elaboración propia con datos del Banco de México

En la Fig. 3, se muestra con la regresión lineal, una relación positiva de las variables, ya que conforme pasa el tiempo el nivel de ingresos por remesas se incrementa, aunque no constantemente, pero sí en las puntuaciones observadas. 


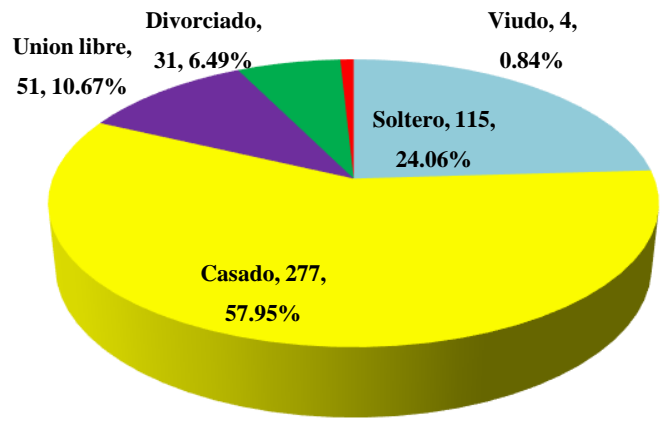

Figura 4 Estado civil

Fuente: Elaboración Propia

En la Fig. 4, con relación al estado civil de los emigrantes, con más del $50 \%$ se encuentra en primer lugar los Casados, siguiendo los solteros, en tercer lugar los que se encuentran Unión libre, posteriormente los Divorciados y por último, los Viudos.

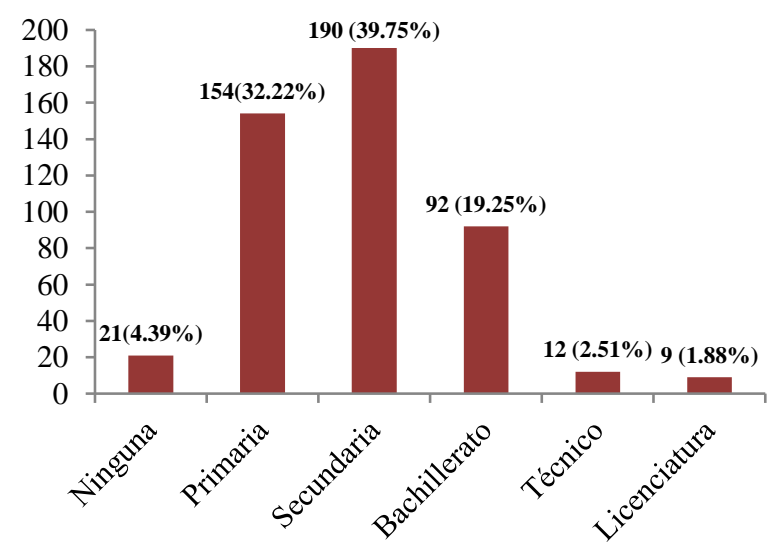

Figura 5 Nivel de educación

Fuente: Elaboración Propia

En la Fig. 5, en relación con el nivel educativo de los emigrantes, se denota que el nivel preponderante es el de secundaria con un $39.75 \%$, con un $32.2 \%$ el nivel primaria y con $19.25 \%$ el bachillerato, como los más representativos, propiciado por la falta de oportunidades mejor pagadas en México comparadas con EUA.

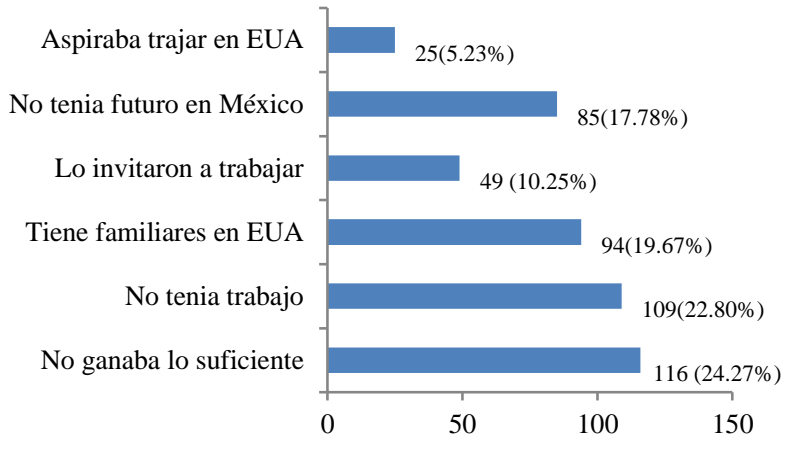

Figura 6 Motivos por los que emigró Fuente: Elaboración Propia

En la Fig. 6, dentro de los motivos que influyen para emigrar se encuentran, principalmente,el no encontrar trabajo en México, y los que hay, no están bien remunerados, así como la falta de oportunidades a futuro, por lo que aprovechan las invitaciones a trabajar en el extranjero, con apoyo de los familiares que se encuentran en EUA.

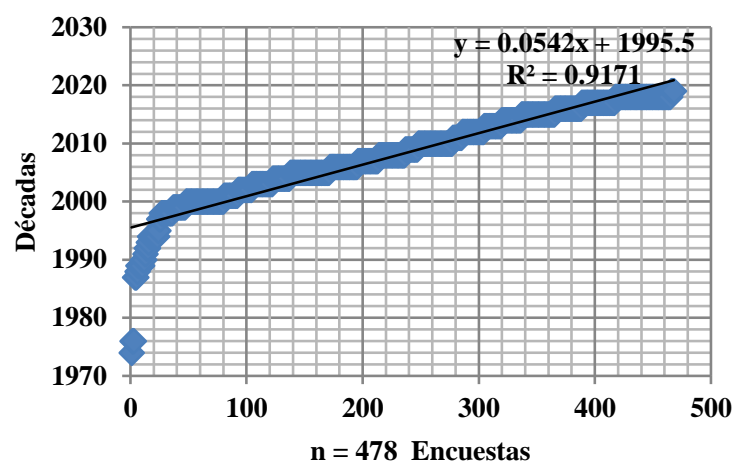

Figura7 Año en que emigró hacia los Estados Unidos Fuente: Elaboración Propia

En la Fig. 7, se muestra que a partir dela década de los 80 's hubo un incremento de la emigración hacia EUA, la cual se mantiene constante de manera ascendiente, pero por otra parte en la década de los noventa a mediados, se presentó una crisis financiera la cual muestra un incremento se presenta un tendencia creciente y en la primera década del siglo XXI en el año 2008 una crisis financiera en Estados Unidos tuvo una repercusión en la economía mexicana, siendo la migración laboral un factor determinante hasta la actualidad, debido a la falta de oportunidades de los mexicanos en su lugar de origen, siendo una alternativa para las familias, mientras que en la segunda década la tendencia no tiene cambio, la ecuación lineal es $\mathrm{y}=0.542 \mathrm{x}+1995.5$, con una pendiente positiva $\mathrm{y}$ $\mathrm{R}^{2}=0.9171$, mostrando una alta correlación . 


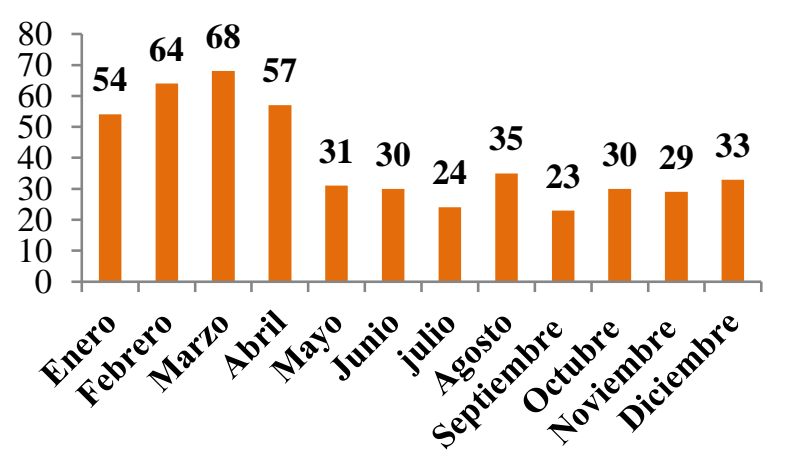

Figura 8 Mes de flujo de emigración hacia Estados Unidos

Fuente: Elaboración Propia

Como lo muestra la Fig. 8 el flujo de emigración es más intenso durante los primeros cuatro meses del año, y disminuye posteriormente, esto se puede deber a la obtención de los recursos para realizar el viaje, o por el clima que propicia la entrada a EUA.

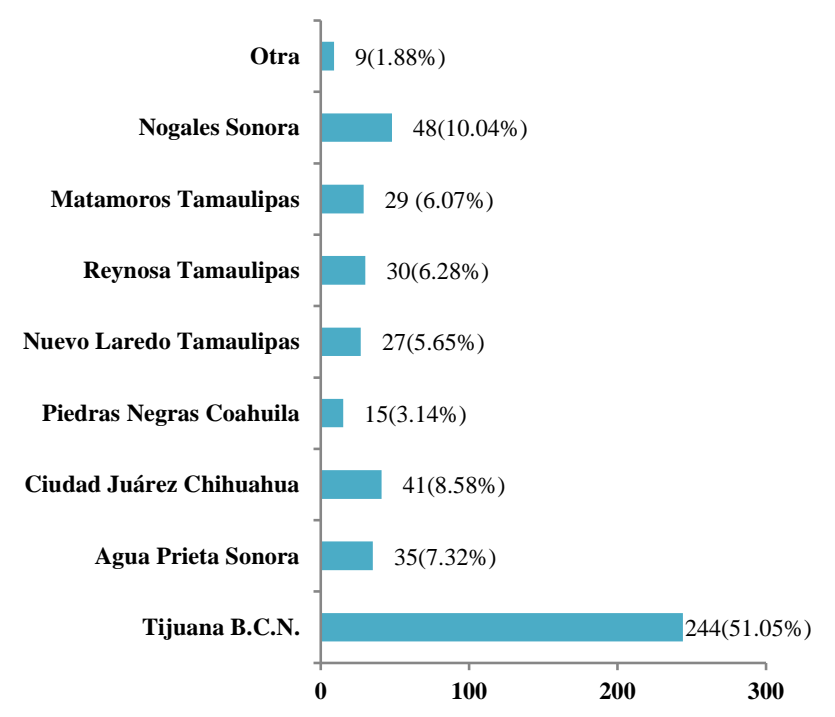

Figura 9 Ciudad fronteriza por la que realizó su cruce a Estados Unidos

Fuente: Elaboración Propia

Como se puede apreciar en la gráfica de la Fig. 9, el lugar que en donde se observa mayor afluencia de emigrantes cruzando la frontera son Tijuana, Baja California Norte, y no solo los connacionales sino también de los centroamericanos, sudamericanos y de otras nacionalidades.De hecho, San Diego y Tijuana comparten "la frontera más cruzada en el mundo", con la gente, la cultura y el comercio fluyendo con facilidad de una ciudad a otra diariamente

(http://www.descubretijuana.com/es/cruzandola-frontera)

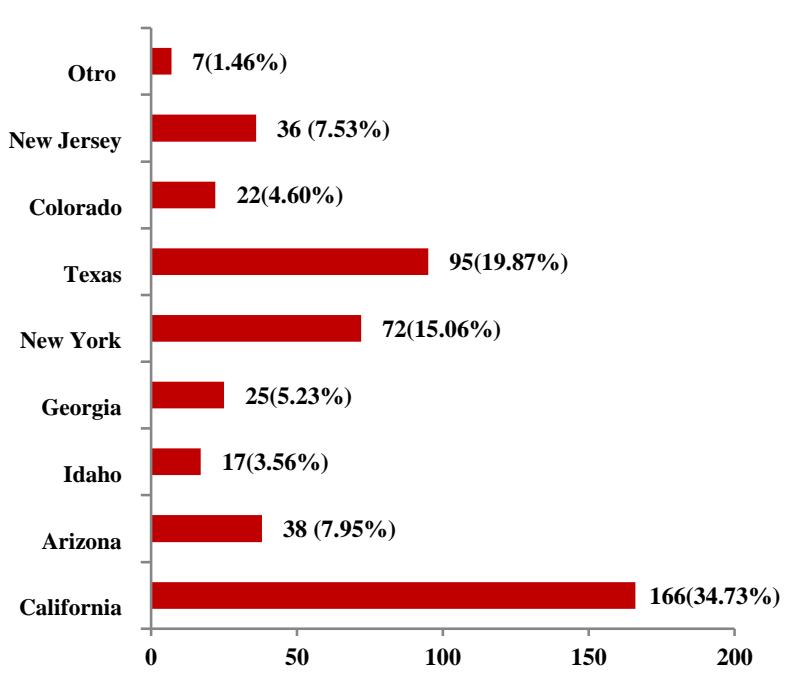

Figura 10 Estado donde residía en los Estados Unidos Fuente: Elaboración Propia

Como se indica en la Fig. 10, el estado que más residen los emigrantes del estudio realizado, es California, de hecho, es el estado donde más habitan migrantes sin documentos en el país: casi una cuarta parte de los 11 millones de personas sin estatus migratorio en todo Estados Unidos. (https://www.univision.com/local/los-angeleskmex/el-mapa-de-los-indocumentados-encalifornia-los-datos-publicos-a-los-que-tieneacceso-ice)

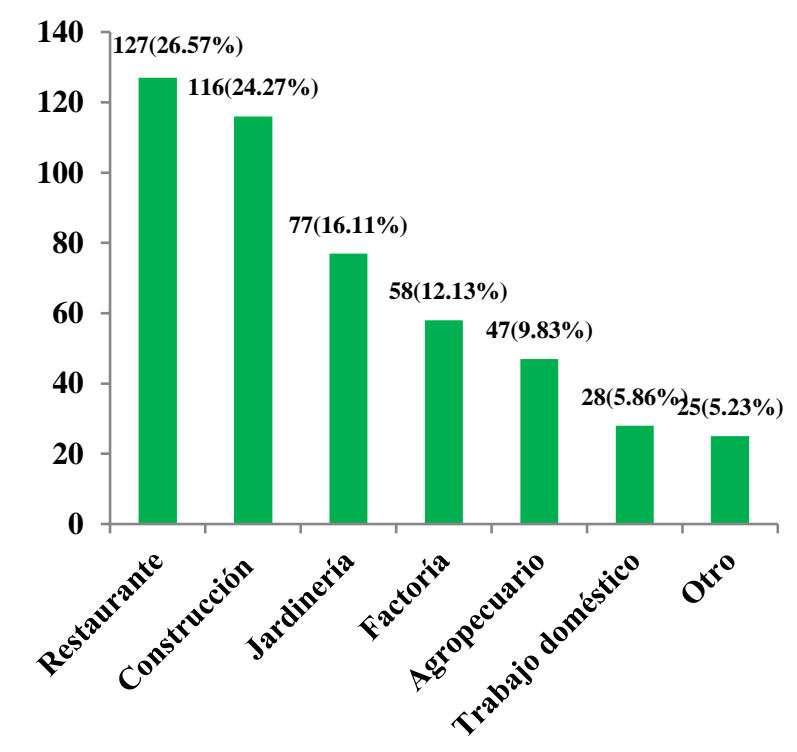

Figura 11 Actividad económica en Estados Unidos Fuente: Elaboración Propia

En la Fig. 11, se denota que las actividades preponderantes son en el sector de restaurantes/lugares de comer o beber, en la construcción y la jardinería. 
En relación a esta información, en 2016, casi una cuarta parte de los empleados de restaurantes eran extranjeros, en comparación con el total de un 18,5 por ciento para todos los sectores, según datos de la Oficina de Estadísticas Laborales recopilados por la Asociación Nacional de Restaurantes.

https://www.nytimes.com/es/2018/12/13/migra ntes-indocumentados-trabajo/

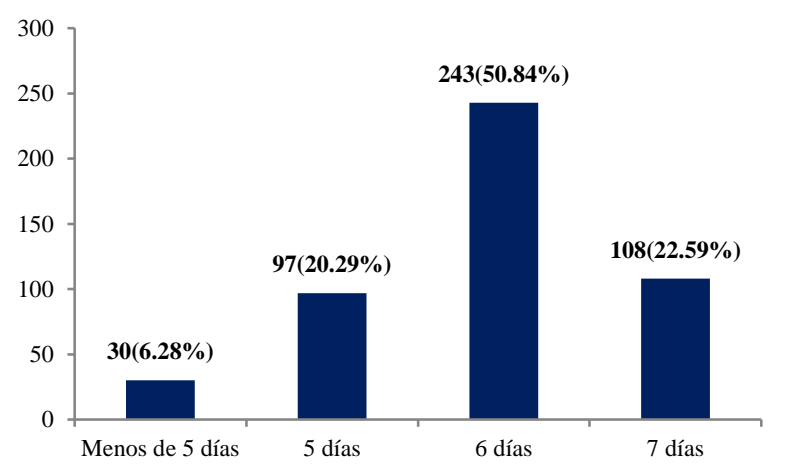

Figura 12 Días por jornada laboral Fuente: Elaboración Propia

En esta Fig. 12, se observa que el 50.84\% de los emigrantes trabajan 6 días a la semana y el $22.59 \%$ toda la semana, esto supone para generar mayores ingresos.

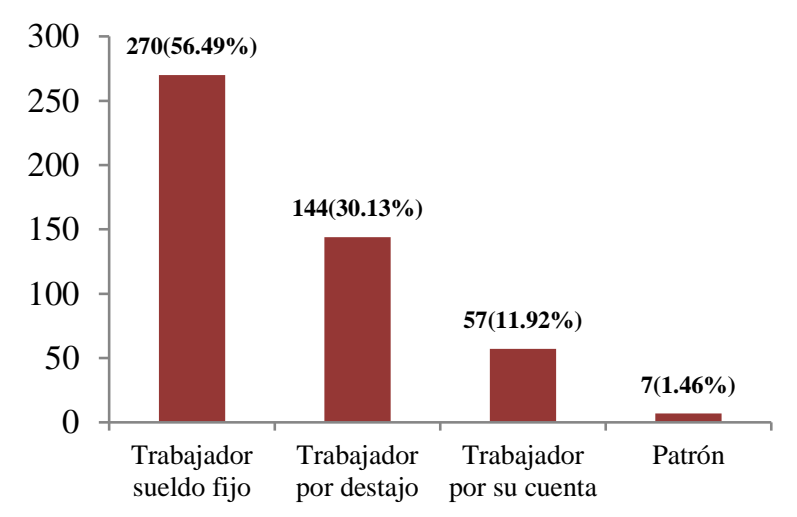

Figura 13 Modalidad de empleo Fuente: Elaboración Propia

Como se ve en la Fig. 13, a la mayoría de los emigrantes les pagan un salario fijo,cifra que alcanza casi el $60 \%, y$,
"Según el estudio Current Population Survey 2015 realizado por el gobierno de Estados Unidos, el 50\% de los hombres y el 25\% de las mujeres inmigrantes provenientes de México, perciben más de 50 mil pesos (USD \$ 2.500) al mes, mientras que en México según el Instituto Nacional de Estadística $y$ Geografía(INEGI) indica que el ingreso promedio para un profesional es de 7.600 pesos (USD \$ 320.00)."

https://es.panampost.com/elena-

toledo/2017/02/27/inmigrantes-mexicanossueldo-eeuu/

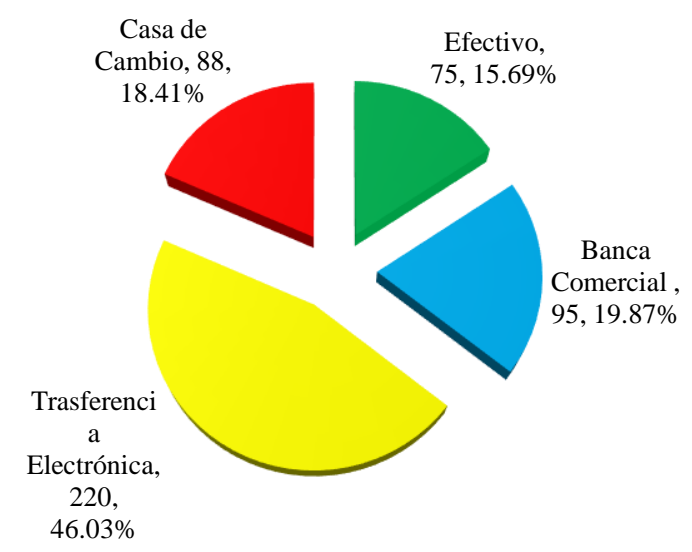

Figura 14 Forma de envío de remesas Fuente: Elaboración Propia

En la Fig. 14 se muestra que el envío de las remesas se da en un $46.03 \%$ en forma electrónica y además,

"El envio de remesas familiares a México en el primer semestre de 2019 alcanzó un total de 16 mil 845 millones de dólares, la cifra más alta para este periodo desde que el Banco de México (Banxico) lleva ese registro en 1995.La cifra es superior a los 16 mil 244 millones de dólares reportados en el primer semestre del año pasado por el Banco Central.Las remesas tuvieron un crecimiento acumulado anual de 6.5 por ciento al cierre del primer semestre de 2019, según los datos ofrecidos por el Banxico."

https://www.elfinanciero.com.mx/economia/re mesas-cierran-primera-mitad-de-2019-conaumento-de-6-5 


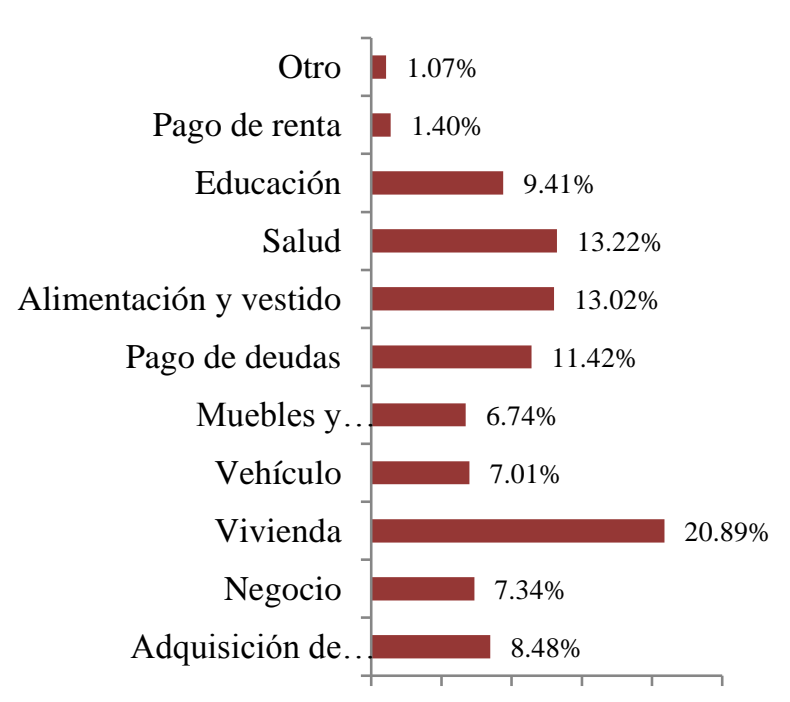

0.005 .0010 .0015 .0020 .0025 .00

Figura 15 Uso de las remesas

Fuente: Elaboración Propia

En la Fig. 15 se observa que el uso de las remesas se designa a la vivienda con un $20.89 \%$ como una inversión a largo plazo. Este es un elemento para decir que la vivienda, así como el consumo en el gasto corriente de las familias en el municipio de Tecamachalco, Puebla son el equivalente de un fondo salarial, si bien Oded Stark, indica que la decisión de emigrar no es sólo una decisión personal, sino que la familia influye en tal decisión por lo que el uso de las remesas es sólo un paliativo, el $13.020 \%$ se destina en alimentación y vestido, el $13.02 \%$ en salud, $11.42 \%$ pago de deudas, $8.48 \%$ adquisición de terrenos, $7.34 \%$ negocio $7.01 \%$ en vehículo, el $1.40 \%$ en pago de la renta y $1.07 \%$ en otro, la migración presenta un multiplicador de la inversión a partir de la inversión y consumo de las familias creando un desarrollo regional.

\section{Conclusiones}

Al presentan factores en que las desigualdades en el grado de desarrollo son más notables, al aumentar la pobreza, cuando las oportunidades de empleo y mejores salarios son mejores en otras regiones o países, o cuando no se respeta la libertad política y religiosa, es cuando las personas deciden emigrar, por lo que se evalúa la alternativa de mejorar, la migración no sólo puede ser laboral, sino que hay diversos tipos de migración: cuando las personas migran en busca de oportunidades de progreso dentro de los límites nacionales, se está en una presencia de migración interna.
Si la migración traspasa fronteras internacionales hablamos de una migración internacional, si la duración es mayor de un año, se considera migrantes permanentes y si es menor a un año, entonces tenemos una migración temporal, otro tipo de migración puede ser que diariamente crucen la frontera para trabajar.

La repercusión económica de la migración en nuestro país se puede delimitar en dos formas: la primera la pérdida de la población en zonas rurales y urbanas en los lugares de origen porque la población que migra principalmente es joven en edad productiva y reproductiva, segunda: las remesas a nivel nacional y familiar, con lo que sus ingresos aumentan y pueden enfrentar una sobrevivencia, a nivel macroeconómico se benefició por el ingreso de divisas, con lo que ayuda a mantener una estabilidad en el tipo de cambio, y la tasa de interés, para estabilizar la economía ante las crisis financieras que han afectado a México.

De acuerdo con la información del Banco de México, las cifras del envío de remesas familiares sólo se encuentran disponibles a nivel nacional y por entidad federativa, pero no se tiene un registro a nivel municipal, es por esto que se tiene que recurrir a los estudios regionales, debido a que los estados no son homogéneos, en el caso de Puebla, por diversos estudios la región que recibe remesas con mayor frecuencia. Las remesas se han convertido en un importante flujo importante de divisas a nivel macroeconómico para la economía mexicana, pero en las economías locales representan un medio de subsistencia, para mejorar las condiciones de vida de las unidades domésticas receptoras, estas remesas sólo han sido un paliativo para contrarrestar los embates de la economía mundial, las disparidades entre regiones al interior de un país y las diferencias entre países, determinan a los agentes económicos, políticos, sociales, financieros, etc.

Los principales destinos de los migrantes de Tecamachalco son: California, Texas y Nueva York, y las ciudades fronterizas con mayor recurrencia son: Tijuana, Nogales, Agua Prieta, Nuevo Laredo, Reynosa y Piedras Negras, las principales actividades que realizaban en los Estados Unidos es el sector servicios (restaurante, ventas, jardinería, transporte) industrial (construcción, factoría) entre otras actividades. 
Los gastos que representan el vivir en los Estados Unidos en cuanto a hospedaje, alimentación, transporte, ropa y calzado, así como otros gastos (teléfono, gas, electricidad) son el alto costo de la vida por lo que las remesas sólo son un ahorro de su ingreso que no podrían obtener en su lugar de origen.

La región de Tecamachalco es una región agrícola, pero también es una región donde confluye una gran variedad de actividades económicas. En la información, se determinó que el principal lugar donde las familias reciben los dólares es en la cabecera municipal de Tecamachalco, donde se ha diversificado los lugares donde reciben las remesas, esto ha permitido un flujo de dólares en el municipio de Tecamachalco por la zona de influencia, económica, política y comercial, los principales días que es notable este fenómeno son los días sábados, debido a que las comunidades utilizan ese día para ir a vender sus productos y adquirir bienes y servicios. La forma en donde se tiene mayor contacto con los flujos de dólares en el municipio de Tecamachalco es por medio de las casas de envío y en menor medida, banca comercial y tiendas departamentales, de conveniencia o farmacias.

En el Directorio Nacional de Unidades Económicas del INEGI (Instituto Nacional de Estadística Geografía e Informática) se contabilizaron 66 unidades económicas con servicios financieros en las cuales las familias que reciben remesas pueden cobrar, por lo que el acceso a la banca de desarrollo ha sido rebasado ante la demanda de tal servicio financiero, por lo que la diversificación se ha desarrollado ante los nuevos cambios, el uso del internet, las redes sociales, no sólo han cambiado a las tecnologías de la información por lo que cada vez más aumenta el número de usuarios en el sector de servicios financieros.

El municipio de Tecamachalco se ha convertido en un nodo de desarrollo, en donde se dinamiza la economía a nivel regional. El multiplicador de la inversión del que Keynes mencionaba era que por parte del gobierno en un supuesto de pleno empleo, tal vez en otra época fue la panacea, sin embargo en la actualidad, los agentes económicos se alejan cada vez más del estado y ahora se toma en cuenta el libre mercado, lo preocupante.
Es que el gobierno debería de realizar diagnósticos regionales para que le permita diseñar políticas públicas a partir de lo local, es necesario identificar las especificaciones de cada región, debido a la diversidad multicultural de México. Otro efecto multiplicador que se presenta en el fenómeno de la migración es que los migrantes sólo envían a sus familias alrededor del $10 \%$ de sus ingresos y el resto dinamiza al país en el cual hayan emigrado, el pago de la renta, alimentación, transporte, salud, lo cual genera un impacto en la economía norteamericana en cuanto consumo e inversión por parte de los migrantes, no sólo como fuerza de trabajo en un mercado laboral internacional.

\section{Referencias}

Aragones, Ana María; Ávila, Sophie; Salgado, Uberto, Migración rural, remesas y su relación con la diversificación sustentable y los patrones de consumo. Un estudio de caso en la zona costera de Oaxaca, Migración y Desarrollo, vol. 14, núm. 27, julio-diciembre, 2016, pp. 109-138. Red Internacional de Migración y Desarrollo. Zacatecas, México.

Arias, Patricia, Migración, economía campesina y ciclo de desarrollo doméstico. Discusiones y estudios recientes, Estudios Demográficos y Urbanos, vol. 28, núm. 1, enero-abril, 2013, pp. 93-121

El Colegio de México, A.C. Distrito Federal, México.

Botello Triana, Jaime, Migración y remesas. Algunos indicadores para México (2000-2008), El Cotidiano, núm. 173, mayo-junio, 2012, pp. 47-55, Universidad Autónoma Metropolitana Unidad Azcapotzalco, Distrito Federal, México.

Canales, Alejandro I.,CANALES, La migración en la reproducción de la sociedad global, Migración y Desarrollo, vol. 11, núm. 21, 2013, pp. 9-4, Red Internacional de Migración y Desarrollo, Zacatecas, México.

Cen Caamal, Marisol, Remesas y estructura del gasto en los hogares receptores: caso de estudio de una comunidad maya contemporánea, Análisis Económico, vol. XXVII, núm. 64, 2012, pp. 5-21 Universidad Autónoma Metropolitana Unidad Azcapotzalco, Distrito Federal, México 
Cuecuecha Mendoza, Alfredo; Adams, Jr., Richard H. El impacto de las remesas sobre tres diferentes indicadores de pobreza en Guatemala, Econo Quantum, vol. 11, núm. 1, 2014, pp. 89112 Universidad de Guadalajara, Zapopan, Jalisco, México.

Chavez Galindo, Ana María, Migraciones internacionales, crisis y vulnerabilidades: Perspectivas comparadas, Migraciones Internacionales, vol. 7, núm. 4, julio-diciembre, 2014, pp. 299-304, El Colegio de la Frontera Norte, A.C. Tijuana, México.

Chavez Arellano, María Eugenia, Experiencias femeninas de migración: Yucatecas en Los Ángeles, Migraciones Internacionales, vol. 7, núm. 4, julio-diciembre, 2014, pp. 69-99, El Colegio de la Frontera Norte, A.C. Tijuana, México.

Corona, Miguel Ángel, Las remesas y el bienestar en las familias de migrantes, Perfiles Latinoamericanos, núm. 43, enero-junio, 2014, pp. 185-207, Facultad Latinoamericana de Ciencias Sociales, Distrito Federal, México.

De la Cruz Gallegos, José Luis; Núñez Mora, José Antonio, Remesas, consumo y desarrollo humano: evidencias de la dependencia de México, Análisis Económico, vol. XXVIII, núm. 67, 2013, pp. 93-121, Universidad Autónoma Metropolitana Unidad Azcapotzalco, Distrito Federal, México.

Díaz González, Eliseo, Reforma migratoria y evolución esperada de las remesas en México Migraciones Internacionales, vol. 7, núm. 2, julio-diciembre, 2013, pp. 245-254, El Colegio de la Frontera Norte, A.C. Tijuana, México.

Duran Jorge y Douglas Massey "Clandestinos, Migración Estados Unidos en los albores del siglo XXI" Universidad Autónoma de Zacatecas y Miguel Ángel Porrúa Editores, México, 2003

Estupian Serrano, Mary Luz, La gestión internacional de las migraciones como una racionalidad política, Migraciones Internacionales, vol. 7, núm. 3, enero-junio, 2014, pp. 249-259

El Colegio de la Frontera Norte, A.C. Tijuana, México.
Figueroa Hernández, Esther; Pérez Soto, Francisco; Godínez Montoya, Lucila, La migración y las remesas en México: 1980-2010, Nóesis. Revista de Ciencias Sociales y Humanidades, vol. 24, núm. 47, enero-junio, 2015, pp. 20-49, Instituto de Ciencias Sociales y Administración, Ciudad Juárez, México.

Fuentes Flores, Noé Arón; González Andrade, Salvador, Medición de las remesas familiares de los migrantes mexicanos en EUA, 1993-2007, Análisis Económico, vol. XXVII, núm. 65, 2012, pp. 151-170, Universidad Autónoma Metropolitana Unidad Azcapotzalco, Distrito Federal, México.

García Gómez, Jazmín; Peláez Herreros, Óscar; Fuentes Flores, Noé Arón, La tradición migratoria como factor explicativo del uso de remesas en la financiación de negocios en Guanajuato, Migraciones Internacionales, vol. 8, núm. 2, julio-diciembre, 2015, pp. 165-193, El Colegio de la Frontera Norte, A.C. Tijuana, México.

García Lourdes G, "La protección del trabajador indocumentado, las legislaciones mexicanas y estadounidense", Tesis, UNAM, México, 1992. Gaspar Olvera, Selene, México Estados Unidos en cifras (1990-2011), Migración y Desarrollo, vol. 10, núm. 18,2012 , pp. 101-139, Red Internacional de Migración y Desarrollo, Zacatecas, México.

Martínez Caballero, Graciela; Montes de Oca Vargas, Hugo, Envejecimiento y migración en los municipios del Estado de México, Papeles de Población, vol. 18, núm. 73, julio-septiembre, 2012, pp. 1-35, Universidad Autónoma del Estado de México, Toluca, México.

Mendoza González, Miguel Ángel; Valdivia López, Marcos, Remesas, crecimiento y convergencia regional en México: aproximación con un modelo Panel especial, Estudios Económicos, vol. 31, núm. 1, enero-junio, 2016, pp. 125-167, El Colegio de México, A.C. Distrito Federal, México.

Maynard Keynes, John "Teoría general de la ocupación el interés y el dinero", Fondo de Cultura Económica, México, 2013. 
Morales, Helda; Aguilar-Stoen, Mariel Cristina; Castellanos-López, Edwin Josué, Migración y remesas: ¿están afectando la sustentabilidad de la agricultura y la soberanía alimentaria en Chiapas?

LiminaR. Estudios Sociales y Humanísticos, vol. XIII, núm. 1, enero-junio, 2015, pp. 29-40, Centro de Estudios Superiores de México y Centro América, San Cristóbal de las Casas, México.

Nava Tablada, Martha Elena, Migración internacional y cafeticultura en Veracruz, México Migraciones Internacionales, vol. 6, núm. 3, enero-junio, 2012, pp. 139-171, El Colegio de la Frontera Norte, A.C. Tijuana, México.

Navarro Chávez, José César Lenin; Rodríguez, José Carlos, Remesas y bienestar social en Michoacán: un enfoque sistémico, Acta Universitaria, vol. 23, núm. 1, noviembre, 2013, pp. 68-75 Universidad de Guanajuato, Guanajuato, México.

Navarro Chávez, José Cesar Lenin; Venegas Martínez, Francisco; Zamora Torres, América Ivonne, Migración y desarrollo económico en México: un análisis factorial de correspondencias, Migración y Desarrollo, vol. 12, núm. 22, enero-junio, 2014, pp. 123-145, Red Internacional de Migración y Desarrollo, Zacatecas, México.

Reyes Morales, Rafael G.; Guijón Cruz, Alicia Sylvia; Cruz Hernández, Isabel, Migración internacional, economías familiares, mercados y medio ambiente en MéxicoMigración y Desarrollo, vol. 13, núm. 25, julio-diciembre, 2015, pp. 117-150, Red Internacional de Migración y Desarrollo, Zacatecas, México.

Salas Alfaro, Renato, La migración internacional y la distribución del ingreso en San Miguel Coatlán, Oaxaca, Estudios Demográficos y Urbanos, vol. 28, núm. 3, septiembre-diciembre, 2013, pp. 711-734, El Colegio de México, A.C., Distrito Federal, México.

Stark Oded, "La migración del trabajo", Ministerio del trabajo y Seguridad Social, España, Madrid España, 1993
Sthephen Castles y Mark J. Miller, "La era de la migración, movimientos internacionales de población en el mundo moderno" Editorial Porrúa, UAZ, Cámara de Diputados, México 2004.

Rojas Soriano, Raúl, Guía para realizar investigaciones sociales, Trigésima octava impresión, Plaza y Valdéz, 2013, México.

Stevenson William J, Estadística para administración y economía", Editorial Harla, 1981, Distrito Federal, México.

Tuirán, Rodolfo; Ávila, José, Migración calificada entre México-Estados Unidos. Desafíos y opciones de política, Migración y Desarrollo, vol. 11, núm. 21, 2013, pp. 43-63, Red Internacional de Migración y Desarrollo, Zacatecas, México. 